\title{
RATIONAL SINGULARITIES AND ALMOST SPLIT SEQUENCES
}

BY

\author{
MAURICE AUSLANDER
}

\begin{abstract}
The main aim of this paper is to relate almost split sequences to singularity theory by showing that the McKay quiver built from the finite-dimensional representations of a finite subgroup $G$ of $G L(2, \mathbf{C})$, where $\mathbf{C}$ is the complex numbers, is isomorphic to the $A R$ quiver of the reflexive modules of the quotient singularity associated with $G$.
\end{abstract}

Over the past decade, almost split sequences have been playing an increasingly important role in the representation theories of finite-dimensional algebras and classical orders (see [5 and 3, 8] for basic existence theorems in these contexts). While they have been known for some time to exist in higher-dimensional situations [3], it has not been at all clear how they related to singularity theory, if at all. The main aim of this paper is to relate almost split sequences to singularity theory by showing that the McKay quiver built from the finite-dimensional complex representations of a finite subgroup $G$ of $\operatorname{GL}(2, \mathbf{C})$, where $\mathbf{C}$ is the complex numbers, is isomorphic to the $A R$ quiver of the reflexive modules over the quotient singularity $R$ associated with $G$. As in the case of finite-dimensional algebras, the $A R$ quiver of reflexive $R$-modules is defined in terms of the almost split sequences of reflexive $R$-modules.

In the case $G \subset \operatorname{SL}(2, \mathbf{C})$, McKay observed that the underlying graph of the McKay quiver, with the trivial module removed, is isomorphic to the desingularization graph of the associated singularity. Various explanations of this phenomenon have been given by Knörrer, Gonzalez-Sprinberg-Verdier [6] and Artin-Verdier [1], which along the way have established, most explicitly in [1], a natural one-to-one correspondence between the indecomposable reflexive $R$-modules and the nodes of the desingularization graph. But why the almost split sequences describe the edge of the desingularization graph still remains to be explained.

An effort has been made to make this paper as self-contained as possible. In particular, no prior knowledge of almost split sequences is required.

Before describing the contents of the six sections of this paper we fix some notation. Throughout this paper $G$ is a finite group, $k$ an algebraically closed field of characteristic not dividing the order of $G$ and $V$ a two-dimensional $k$-representation of $G$. Setting $S=k[[X, Y]]$, the $k$-algebra of formal power series, the two-dimensional representation $V$ gives a linear action of $G$ on $S$ as a group of $k$-algebra automorphisms. We denote by $S[G]$ the skew group ring given by this action.

Received by the editors January 2, 1985.

1980 Mathematics Subject Classification. Primary 14J17.

'Partially supported by NSF Grant \# MCS 8303348.

(1986 American Mathematical Society $0002-9947 / 86 \$ 1.00+\$ .25$ per page 
$\S 1$. In this section the McKay quiver of $G$ with respect to $V$ is defined and shown to be given by the minimal $S[G]$-projective resolutions of the simple $S[G]$-modules.

$\S 2$. This section is devoted to showing that the minimal $S[G]$-projective resolutions of simple $S[G]$-modules are essentially the same thing as the almost split sequences in the category of reflexive modules of the quotient singularity $R=S^{G}$ in case $V$ is a faithful $G$-module having no pseudo-reflections. This is used to show that the McKay quiver of $G$ with respect to $V$ is isomorphic to the $A R$ quiver of the reflexive $R$-modules.

$\S 3$. We continue with the hypothesis that $V$ is a faithful $G$-module having no pseudo-reflections and show how the almost split sequences for the reflexive $R$-modules can be constructed from a fixed exact sequence of reflexive $R$-modules called the fundamental exact sequence.

$\S 4$. In this section we assume that $k$ is $\mathbf{C}$, the complex numbers. We give a different proof of the following result in Artin-Verdier [1]. The $\mathbf{C}$-algebras which are integrally closed, complete, local two-dimensional noetherian domains with residue class field $\mathbf{C}$ having only a finite number of nonisomorphic reflexive indecomposable modules are precisely the two-dimensional quotient singularities.

$\S 5$. In this section the notion of reflexive splitting trace modules over arbitrary integrally closed noetherian domains is introduced and some elementary properties established.

§6. In this the last section of the paper, we use the notion of reflexive splitting trace modules to generalize the results of $\S 3$ about the construction of almost split sequences from the fundamental exact sequences to arbitrary complete, integrally closed two-dimensional noetherian local domains.

It should be noted that the results of $\$ \S 5$ and 6 have almost exact analogues in the theory of modular group representations [4].

Finally, I would like to thank M. Artin, R. Buchweitz, H. Knörrer, I. Reiten and G. Schwarz, for many helpful conversations.

1. The McKay quiver and projective resolutions. We assume throughout this section that $G$ is a finite group and $k$ a field of characteristic not dividing the order of $G$. Then $k[G]$, the group ring of $G$ over $k$, is semisimple and we denote by $V_{0}$, $V_{1}, \ldots, V_{d}$ a complete set of nonisomorphic simple $k[G]$-modules with $V_{0} \simeq k$, the trivial representation. Let $V$ be a fixed two-dimensional $k[G]$-module. We recall that the McKay quiver of $V$ is the directed graph with vertices the $V_{0}, \ldots, V_{d}$ and having $m$ arrows from $V_{i}$ to $V_{j}$ if and only if the multiplicity of $V_{i}$ in $V \otimes_{k} V_{j}$ is $m$. Our aim in this section is to interpret the McKay quiver in terms of the projective resolutions of simple modules over certain skew group rings.

Let $S=k[[X, Y]]$ be the formal power series in two variables over $k$. Then the two-dimensional representation $V$ induces a linear action of $G$ on $S$ as a group of $k$-automorphisms. We recall that the skew group $\operatorname{ring} S[G]$ of $G$ over $S$ is additively the free $S$-module with basis the elements of $G$ and with multiplication given by $\left(s_{1} \sigma_{1}\right)\left(s_{2} \sigma_{2}\right)=s_{1} \sigma_{1}\left(s_{2}\right) \sigma_{1} \sigma_{2}$, where the $s_{i}$ are in $S$ and the $\sigma_{i}$ are in $G$ for $i=1,2$. An $S[G]$-module is an $S$-module $M$ together with a $G$-module structure satisfying 
$\sigma(s m)=\sigma(s) \sigma(m)$ for all $\sigma$ in $G, s$ in $S$ and $m$ in $M$. Also, if $M_{1}$ and $M_{2}$ are $S[G]$-modules, then the morphisms from $M_{1}$ to $M_{2}$ consist of the $S$-module morphisms which are also $G$-module morphisms.

Next we observe that if $M$ and $N$ are $S[G]$-modules, then $\operatorname{Hom}_{S}(M, N)$ will be considered an $S[G]$-module by means of the usual action of $G$ on $\operatorname{Hom}_{S}(M, N)$ given by $(\sigma f)=\sigma\left(f\left(\sigma^{-1} m\right)\right)$ for all $\sigma$ in $G, f$ in $\operatorname{Hom}_{S}(M, N)$ and $m$ in $M$. As usual, we have that $\operatorname{Hom}_{S[G]}(M, N)=\operatorname{Hom}_{S}(M, N)^{G}$, the fixed points of the action of $G$ on $\operatorname{Hom}_{S}(M, N)$. An easy consequence of these remarks is the following useful criterion for when an $S[G]$-module is projective.

Lemma 1.1. An $S[G]$-module $X$ is projective if and only if it is a free $S$-module.

Proof. Since $S[G]$ is a free $S$-module, any projective $S[G]$-module is a projective and therefore free $S$-module.

Suppose $X$ is an $S[G]$-module which is a free $S$-module. Let $0 \rightarrow A \rightarrow B \rightarrow C \rightarrow 0$ be an exact sequence of $S[G]$-modules. Then $0 \rightarrow \operatorname{Hom}_{S}(X, A) \rightarrow \operatorname{Hom}_{S}(X, B) \rightarrow$ $\operatorname{Hom}_{S}(X, C) \rightarrow 0$ is an exact sequence of $S[G]$-modules. Since $k[G]$ is semisimple, the fixed point functor is exact, so $\operatorname{Hom}_{S[G]}(X, B) \rightarrow \operatorname{Hom}_{S[G]}(X, C) \rightarrow 0$ is exact. This shows that $X$ is $S[G]$-projective.

Our next aim is to use Lemma 1.1 to obtain an explicit description of the projective $S[G]$-modules which will be useful in describing the projective resolutions of the simple $S[G]$-modules. The first step in this direction is based on the following definitions.

Let $M$ be an $S[G]$-module and $W$ a $k[G]$-module. We consider $M \otimes_{k} W$ an $S[G]$-module by means of the operation $s \sigma(m \otimes w)=s \sigma(m) \otimes \sigma(w)$ for all $s$ in $S, \sigma$ in $G, m$ in $M$ and $w$ in $W$. Also, we denote by $S$ the projective $S[G]$-module which is the $S$-module $S$ together with our given operation of $G$ on $S$. As an easy consequence of these definitions and Lemma 1.1 we have the following

LEMMA 1.2. If $P$ is a projective $S[G]$-module, then $P \otimes_{k} W$ is a projective $S[G]$-module for all $k[G]$-modules $W$. In particular, $S \otimes_{k} W$ is a projective $S[G]$-module for all $k[G]$-modules $W$.

Proof. It follows from the definition of $P \otimes_{k} W$, that $P \otimes_{k} W$ is $S$-free and therefore $S[G]$-projective.

We now show that all projective $S[G]$-modules can be written uniquely up to isomorphism in the form $S \otimes_{k} W$ with $W$ in $\bmod k[G]$.

Let $m$ be the maximal ideal $(X, Y)$ of $S$. Since $\sigma(m)=m$ for all $\sigma$ in $G$, it follows that $\mathrm{m} S[G]$ is a two-sided ideal in $S[G]$. This implies that $\mathrm{m} S[G]$ is contained in the radical of $S[G]$ since $m$ is the radical of $S$ and $S[G]$ is a finitely generated $S$-module. From this it follows that $\mathrm{m} S[G]$ is the radical of $S[G]$ since $S[G] / \mathrm{m} S[G] \simeq k[G]$ which is semisimple. Thus, if $P$ is a projective $S[G]$-module, then $P / \mathrm{m} P$ is a $k[G]$-module. Moreover, since $S$ is complete, the natural epimorphism $P \rightarrow P / \mathrm{m} P$ is a projective cover. Consequently, two projective $S[G]$-modules $P$ and $Q$ are isomorphic if and only if $P / \mathrm{m} P$ is isomorphic to $Q / \mathrm{m} Q$. As a consequence of these 
remarks we have the following

Proposition 1.3. (a) If $P$ is a projective $S[G]$-module, then $P \simeq S \otimes_{k} P / m P$.

(b) If the projective $S[G]$-modules $S \otimes_{k} W$ and $S \otimes_{k} W^{\prime}$ are isomorphic, then the $k[G]$-modules $W$ and $W^{\prime}$ are isomorphic.

Proof. Follows from previous observations and the fact that $S \otimes_{k} W$ is the projective cover of $S / \mathrm{m} \otimes_{k} W \simeq W$ for all $k[G]$-modules $W$.

As an immediate consequence of Proposition 1.3, we have the following

COROllary 1.4. Let $V_{0}, V_{1}, \ldots, V_{d}$ be a complete set of nonisomorphic simple $k[G]$-modules. Then $S \otimes_{k} V_{0}, \ldots, S \otimes_{k} V_{d}$ is a complete set of nonisomorphic indecomposable projective $S[G]$-modules.

We are now ready to describe the projective resolutions of the simple $S[G]$-modules. We begin with the simple $S[G]$-module $k$ with trivial group action. Since $S=k[[X, Y]]$, we know that the minimal projective $S$-resolution $0 \rightarrow S \rightarrow S \amalg S \rightarrow$ $S \rightarrow S / \mathrm{m} \rightarrow 0$ is given by the Koszul complex which can be written as $0 \rightarrow S$ $\otimes_{k} \wedge^{2} V \rightarrow S \otimes_{k} V \rightarrow S \rightarrow k \rightarrow 0$ where $V$ has been identified with $k X \amalg k Y$. But it can easily be checked that the morphisms involved are also $G$-morphisms where $G$ acts on the modules involved as defined above. Therefore this sequence is also a minimal projective $S[G]$-resolution of $k$. Now to obtain the minimal projective $S[G]$-resolution of an arbitrary simple $S[G]$-module $V_{j}$, we simply tensor the Koszul complex with $V_{j}$ over $k$, getting

$$
0 \rightarrow S \otimes_{k}\left(\wedge^{2} V \otimes_{k} V_{j}\right) \rightarrow S \otimes_{k}\left(V \otimes_{k} V_{j}\right) \rightarrow S \otimes_{k} V_{j} \rightarrow V_{j} \rightarrow 0
$$

This shows that the McKay quiver for $V$ is isomorphic to the following quiver $\Gamma$ associated with $S[G]$ in analogy with the situation for artin algebras.

Let $P_{i}$ be a $S[G]$-projective cover of the simple $S[G]$-module $V_{i}$. Then $P_{0}, \ldots, P_{d}$ is a complete set of nonisomorphic indecomposable projective $S[G]$-modules which we consider the vertices of $\Gamma$. For each $j=0, \ldots, d$, let

$$
0 \rightarrow Q_{2}^{(j)} \rightarrow Q_{1}^{(j)} \rightarrow Q_{0}^{(j)} \rightarrow V_{j} \rightarrow 0
$$

be a minimal projective resolution of $V_{j}$. Then the number of arrows from $P_{i}$ to $P_{j}$ in $\Gamma$ is the multiplicity of $P_{i}$ in a decomposition into indecomposables of $Q_{1}^{(j)}$. Denote this quiver by $\Gamma(V)$. Clearly, we have an isomorphism of $\Gamma(V)$ with the McKay quiver of $V$ by sending $P_{i}$ to $V_{i}$ for all $i=0, \ldots, d$.

2. McKay quivers and $A R$ quivers. In addition to the hypothesis of $\S 1$, we assume $k$ is an algebraically closed field and that the two-dimensional $k[G]$-module $V$ has no pseudo-reflections, i.e. no $\sigma \neq\{1\}$ in $G$ has 1 as an eigenvalue. Then the invariant algebra $R=S^{G}$ is a complete, integrally closed, two-dimensional domain and $S$ is a finitely generated $R$-module. The hypothesis that $G$ has no pseudo-reflections in $V$ is equivalent to the height one primes in $R$ being unramified in $S$ (cf. [2]). Our aim in this section is to give a description of the quiver $\Gamma(V)$, and therefore of the McKay quiver, in terms of the almost split sequences in the category $\mathfrak{R}$ of reflexive $R$-modules. 
Recall that an $R$-module $M$ is said to be reflexive if the natural morphism $M \rightarrow \operatorname{Hom}_{R}\left(\operatorname{Hom}_{R}(M, R), R\right)$ is an isomorphism. Since $R$ is integrally closed of dimension two, it follows that the reflexive $R$-modules are the Cohen-Macaulay $R$-modules. Hence $S$ is a reflexive $R$-module. We denote by add $S$ the full subcategory of $\mathfrak{Z}$ consisting of the summands of finite sums of $S$. Clearly, add $S$ is an additive category in which idempotents split. For the sake of completeness, we prove the following well known fact [7]:

Proposition 2.1. Up to isomorphism, the indecomposable reflexive $R$-modules are precisely the indecomposable $R$-summands of $S$. Hence $\mathfrak{R}=$ add $S$ has only a finite number of nonisomorphic indecomposable reflexive $R$-modules.

Proof. Let $L$ be an indecomposable reflexive $R$-module. The fact that the characteristic of $k$ is relatively prime to the order of $G$ implies that the inclusion of $R$-modules $0 \rightarrow R \rightarrow S$ splits. Therefore, we have that the monomorphism of $R$-modules $0 \rightarrow \operatorname{Hom}_{R}\left(M^{*}, R\right) \rightarrow \operatorname{Hom}_{R}\left(M^{*}, S\right)$ also splits, where $M^{*}=$ $\operatorname{Hom}_{R}(M, R)$. Since $\operatorname{Hom}_{R}\left(M^{*}, S\right)$ is a Cohen-Macaulay $S$-module, it is a free $S$-modules, so $M=M^{* *}$ is a summand of a finite sum $n S$ as $R$-modules. Because $R$ is complete, $\bmod R$ is a Krull-Schmidt category. Hence, the fact that $L$ is indecomposable and a summand of a finite sum of copies of $S$ implies that $L$ is a summand of $S$. The rest of the proposition is trivial.

Our aim now is to describe an equivalence of categories between $\mathfrak{B}$, the category of projective $S[G]$-modules and $\mathfrak{Z}=\operatorname{add}_{R} S$, the category of reflexive $R$-modules.

Straightforward calculations show that the $R$-submodule $S[G]^{G}$ of $S[G]$ consists of all elements of the form $\sum_{\sigma \in G} \sigma(s) \sigma$ with $s$ in $S$. Thus, we obtain the $R$-module isomorphism $S \rightarrow S[G]^{G}$ given by $s \mapsto \sum_{\sigma \in G} \sigma(s) \sigma$ for all $s$ in $S$ which we view as an identification. Therefore, $P^{G}$ is in $\operatorname{add}_{R} S$ for all $P$ in $\mathfrak{P}$, and so the functor $\bmod S[G] \rightarrow \bmod R$ given by $A \mapsto A^{G}$ induces a functor $\mathfrak{B} \rightarrow \operatorname{add}_{R} S$ which we now show is an equivalence of categories.

First of all it is easy to see that $\mathfrak{B} \rightarrow \operatorname{add}_{R} S$ is an equivalence if and only if the $R$-algebra morphism $\operatorname{End}_{S[G]}(S[G]) \rightarrow \operatorname{End}_{R}(S)$ given by $f \mapsto f \mid S$ for all $f$ in $\operatorname{End}_{S[G]}(S[G])$ is an isomorphism. Next we have the $R$-algebra isomorphism $\beta$ : $S[G]^{\text {op }} \rightarrow \operatorname{End}_{S[G]}(S[G])$ given by $\beta(s \sigma)(x)=x \cdot s \sigma$ for all $s$ in $S$, $\sigma$ in $G$ and $x$ in $S[G]$. Also, we have the $R$-algebra isomorphism $S[G] \rightarrow S[G]^{\text {op }}$ given by $s \sigma \mapsto$ $\sigma^{-1}(s) \sigma^{-1}$ for all $s$ in $S$ and $\sigma$ in $G$. Finally, it is straightforward to check that the composition of $R$-algebra morphisms

$$
S[G] \rightarrow S[G]^{\mathrm{op}} \stackrel{\beta}{\rightarrow} \operatorname{End}_{S[G]}(S[G]) \rightarrow \operatorname{End}_{R}(S)
$$

is the standard $R$-algebra morphism $\gamma: S[G] \rightarrow \operatorname{End}_{R}(S)$ given by $\gamma(s \sigma)(x)=s \sigma(x)$ for all $s$ in $S, \sigma$ in $G$ and $x$ in $S$. Hence we have that $\gamma: S[G] \rightarrow \operatorname{End}_{R}(S)$ is an isomorphism if and only if $\operatorname{End}_{S[G]}(S[G]) \rightarrow \operatorname{End}_{R}(S)$ is an isomorphism if and only if $\mathfrak{P} \rightarrow \operatorname{add}_{R} S$ is an equivalence. But the fact that the height one primes in $R$ are unramified in $S$ implies that $\gamma: S[G] \rightarrow \operatorname{End}_{R}(S)$ is an isomorphism [2]. 
Therefore, we have established the following

Proposition 2.2. The functor $\mathfrak{B} \rightarrow \mathfrak{Q}=\operatorname{add}_{R} S$ given by $P \mapsto P^{G}$ for $P$ in $\mathfrak{B}$ is an equivalence of categories.

Our aim now is to apply this proposition to construct in terms of the almost split sequences in $\&$ a quiver which is isomorphic to the quiver $\Gamma(V)$ defined in the previous section. To this end it is convenient to introduce some notation. As usual, let $V_{0}, \ldots, V_{d}$ be a complete set of nonisomorphic simple $S[G]$-modules with $V_{0}=k$, the trivial simple, and let $P_{i}$ be a projective cover for $V_{i}$ for all $i=0, \ldots, d$ with $P_{0}=S$. Then $P_{0}, \ldots, P_{d}$ is a complete set of nonisomorphic indecomposable projective $S[G]$-modules. Furthermore, we know that $P_{i} \simeq S \otimes_{k} V_{i}$ for all $i=0, \ldots, d$. Also we obtained in the previous section the minimal projective resolutions

$$
0 \rightarrow S \otimes_{k}\left(\stackrel{2}{\Lambda} \otimes_{k} V_{i}\right) \rightarrow S \otimes_{k}\left(V \otimes_{k} V_{i}\right) \rightarrow S \otimes_{k} V_{i} \rightarrow V_{i} \rightarrow 0 .
$$

Since $\wedge^{2} V$ is of dimension 1 over $k$, we have $\wedge^{2} V \otimes_{k} V_{i}$ is a simple $k[G]$-module which we denote by $\tau\left(V_{i}\right)$. Clearly, $\tau\left(V_{i}\right) \simeq \tau\left(V_{j}\right)$ if and only if $V_{i} \simeq V_{j}$. Therefore, $S \otimes_{k} \tau\left(V_{i}\right)$ is an indecomposable projective $S[G]$-module. We define $\tau\left(P_{i}\right)$ to be the unique $P_{j}$ isomorphic to $S \otimes_{k}\left(V_{i}\right)$. Then we can write the minimal projective resolutions of the $V_{i}$ as

$$
0 \rightarrow \tau\left(P_{i}\right) \stackrel{u_{i}}{\rightarrow} Q_{i} \stackrel{v_{i}}{\rightarrow} P_{i} \rightarrow V_{i} \rightarrow 0
$$

where $Q_{i} \simeq S \otimes_{k}\left(V \otimes_{k} V_{i}\right)$. Now denoting $P_{L}^{G}, Q_{L}^{G}$ and $\tau\left(P_{i}\right)^{G}$ by $L_{i}, E_{i}$ and $\tau\left(L_{i}\right)$, respectively, we have that the $L_{0}, \ldots, L_{d}$ are a complete set of nonisomorphic indecomposable reflexive $R$-modules and so are the $\tau\left(L_{0}\right), \ldots, \tau\left(L_{d}\right)$.

Applying the fixed point functor to the exact sequence

$$
0 \rightarrow \tau\left(P_{0}\right) \rightarrow Q_{0} \stackrel{u_{0}}{\rightarrow} P_{0} \stackrel{v_{0}}{\rightarrow} k \rightarrow 0,
$$

we obtain the exact sequence of $R$-modules

$$
0 \rightarrow \tau(R) \stackrel{g_{0}}{\rightarrow} E_{0} \stackrel{f_{0}}{\rightarrow} R \rightarrow k \rightarrow 0 .
$$

We first observe that $f_{0}: E_{0} \rightarrow R$ has the property that if a morphism $h: L \rightarrow R$ is not a splittable epimorphism then it can be lifted to $E_{0}$, i.e. there is $j: L \rightarrow E_{0}$ such that $f_{0} j=h$. To see this we use the equivalence $\mathfrak{B} \rightarrow \mathfrak{L}$. Let $h_{p}: P \rightarrow S$ be a morphism in $\mathfrak{P}$ such that $h_{p}^{G}: P^{G} \rightarrow S^{G}$ is the morphism $h: L \rightarrow R$. Since $h$ is not a splittable epimorphism, $h_{p}$ is not a splittable epimorphism. Therefore, $\operatorname{Im} h_{p} \subset \mathrm{m} S$ $=\operatorname{Im} v_{0}$, which means that $h_{p}$ can be lifted to $v_{0}$. Hence $h$ can be lifted to $E_{0}$.

We now show that if

$$
0 \rightarrow A \stackrel{g}{\rightarrow} B \stackrel{f}{\rightarrow} R \rightarrow k \rightarrow 0
$$

is an exact sequence of $R$-modules with $A, B$ and $R$ in 2 with $A$ indecomposable having the property that any morphism $h: L \rightarrow R$ in $\mathfrak{L}$ which is not a splittable epimorphism can be lifted to $B$, then $0 \rightarrow A \rightarrow B \rightarrow R \rightarrow k \rightarrow 0$ is isomorphic to 
$0 \rightarrow \tau(R) \rightarrow E_{0} \rightarrow R \rightarrow k$. For by definition we have a commutative diagram

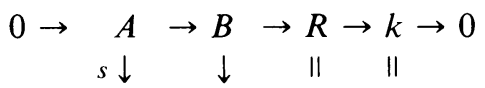

$$
\begin{aligned}
& 0 \rightarrow \tau(R) \rightarrow E_{0} \rightarrow R \rightarrow k \rightarrow 0
\end{aligned}
$$

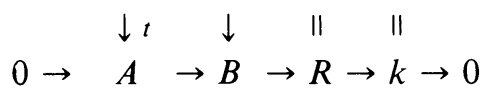

Now the endomorphism $\operatorname{Ext}^{2}(k, t s): \operatorname{Ext}^{2}(k, A) \rightarrow \operatorname{Ext}^{2}(k, A)$ induced by the endomorphism $t s: A \rightarrow A$ has the property that $\operatorname{Ext}^{2}(k, t s)(x)=x$, where $x$ is the nonzero element $0 \rightarrow A \rightarrow B \rightarrow R \rightarrow k \rightarrow 0$ in $\operatorname{Ext}^{2}(k, A)$. Therefore by Nakayama's lemma, $t s$ is not in the radical of $\operatorname{End}_{R}(A)$ and therefore is an isomorphism since $\operatorname{End}_{R}(A)$ is a local ring. Therefore, $s: A \rightarrow \tau(R)$ is an isomorphism since $\tau(R)$ is indecomposable, showing that $0 \rightarrow A \rightarrow B \rightarrow R \rightarrow k \rightarrow 0$ is isomorphic to $0 \rightarrow \tau(R) \rightarrow E_{0} \rightarrow R \rightarrow k \rightarrow 0$. This uniquely determined exact sequence $0 \rightarrow \tau(R) \stackrel{v_{0}}{\rightarrow} E_{0} \stackrel{u_{0}}{\rightarrow} R \rightarrow k \rightarrow 0$ in $\bmod R$ is called the fundamental exact sequence.

Suppose now that $V_{i} \neq k$. Then $V_{i}^{G}=0$. Hence applying the fixed point functor to the exact sequence

$$
0 \rightarrow \tau\left(P_{i}\right) \stackrel{u_{i}}{\rightarrow} Q_{i} \stackrel{v_{i}}{\rightarrow} P_{i} \rightarrow V_{i} \rightarrow 0
$$

we obtain the exact sequence of reflexive $R$-modules

$$
0 \rightarrow \tau\left(L_{i}\right) \stackrel{g_{i}}{\rightarrow} E_{i} \stackrel{f_{i}}{\rightarrow} L_{i} \rightarrow 0
$$

Obviously $f_{i}$ is not a splittable epimorphism. Using an argument similar to the argument used to characterize the fundamental exact sequence, it is not difficult to show that an exact sequence $0 \rightarrow A \rightarrow B \rightarrow L_{i} \rightarrow 0$ with $A$ and $B$ in $\mathfrak{Q}$ is isomorphic to $0 \rightarrow \tau\left(L_{i}\right) \stackrel{g_{i}}{\rightarrow} E_{i} \stackrel{f_{i}}{\rightarrow} L_{i} \rightarrow 0$ if and only if it satisfies the following conditions:

(a) $A$ is indecomposable;

(b) it does not split;

(c) every morphism $h: L \rightarrow L_{i}$ in $\mathfrak{Q}$ which is not a splittable epimorphism can be lifted to $B$.

Thus for each $L_{i} \neq R$ the exact sequence $0 \rightarrow \tau\left(L_{i}\right) \rightarrow E_{i} \rightarrow L_{i} \rightarrow 0$ is uniquely determined by $L_{i}$ and is called the almost split sequence determined by $L_{i}$. Following the procedure used in the theory of artin algebras we define for $R$ the following quiver, usually called the $A R$ quiver of $\mathfrak{Z}$ which we will denote by $A R(\Omega)$.

The vertices of the $A R$ quiver of $\mathfrak{Z}$ are the indecomposable reflexive $R$-modules $L_{0}, L_{1}, \ldots, L_{d}$ and the number of arrows from $L_{j}$ to $L_{i}$ is the multiplicity of $L_{j}$ in a decomposition of $E_{i}$ into a sum of indecomposable modules. It is obvious that by construction there is an isomorphism $A R(\mathfrak{R}) \rightarrow \Gamma(V)$ sending $L_{i}$ to $P_{i}$ for all $i$. Combining this isomorphism with the isomorphism between $\Gamma(V)$ and the McKay quiver of $V$ already described in $\S 1$, we obtain an isomorphism between the $A R$ and McKay quivers. 
3. Construction of almost split sequences. We continue with the same hypothesis and notation as in the previous section. Our main aim in this section is to show how all the almost split sequences in $\{$ can be derived from the fundamental exact sequence in a rather simple way. It is for this reason that the exact sequence $0 \rightarrow \tau(R) \rightarrow E_{0} \rightarrow R \rightarrow k \rightarrow 0$ is called the fundamental exact sequence. We begin with some preliminaries.

Since $R$ is a complete local ring, we know that it contains a complete regular local ring $T$ of dimension 2 such that $R$ is a finitely generated $T$-module. In [3] it was shown that $\tau(R)=\operatorname{Hom}_{T}(R, T)$. Therefore, $\tau(R)=w$, the dualizing $R$-module. Therefore, $\operatorname{Ext}_{R}^{2}(k, w) \simeq k$ and the fundamental exact sequence is any one of the nonzero elements in $\operatorname{Ext}_{R}^{2}(k, w)$. We will denote $\tau(R)$ by $w$ from now on.

Now given two $S\left[G\right.$ ]-modules $A$ and $B$ we consider the $S$-module $A \otimes_{S} B$ given by $\sigma(a \otimes b)=\sigma(a) \otimes \sigma(b)$ for all $\sigma$ in $G, a$ in $A$ and $b$ in $B$. It is easily seen that for all $A, B$ and $C$ in mod $S[G]$ we have the $S[G]$-isomorphisms

$$
\operatorname{Hom}_{S}\left(A \otimes_{S} B, C\right) \rightarrow \operatorname{Hom}_{S}\left(A, \operatorname{Hom}_{S}(B, C)\right)
$$

which are functorial in $A, B$ and $C$. Applying the $G$-fixed point functor we have functorial isomorphism

$$
\operatorname{Hom}_{S[G]}\left(A \otimes_{S} B, C\right) \rightarrow \operatorname{Hom}_{S[G]}\left(A, \operatorname{Hom}_{S}(B, C)\right) .
$$

Now if $P$ and $Q$ are $S[G]$-projective, then $P \otimes_{S} Q$ is $S$-free and hence $S[G]$-projective. Since

$$
S / \mathfrak{m}\left(P \otimes_{S} Q\right) \simeq P / \mathfrak{m} P \otimes_{k} Q / \mathrm{m} Q
$$

the functor $\mathfrak{B} \rightarrow \bmod k[G]$ commutes with tensor products. Also we have that $\left(S \otimes_{k} W_{1}\right) \otimes_{S}\left(S \otimes_{k} W_{2}\right) \simeq S \otimes_{k}\left(W_{1} \otimes_{k} W_{2}\right)$ for all $W_{1}$ and $W_{2}$ in $\bmod k[G]$. Therefore the projective $S[G]$-resolutions

$$
0 \rightarrow S \otimes_{k}\left(\bigwedge^{2} V \otimes_{k} V_{j}\right) \rightarrow S \otimes_{k}\left(V \otimes_{k} V_{j}\right) \rightarrow S \otimes_{k} V_{j} \rightarrow V_{j} \rightarrow 0
$$

can be rewritten as

$$
0 \rightarrow\left(S \otimes_{k} \wedge V\right) \otimes_{S}\left(S \otimes_{k} V_{j}\right) \rightarrow\left(S \otimes_{k} V\right) \otimes_{S}\left(S \otimes_{k} V_{j}\right) \rightarrow S \otimes_{k} V_{j} \rightarrow V_{j} \rightarrow 0
$$

and therefore as

$$
0 \rightarrow \tau(S) \otimes_{S} P_{j} \rightarrow Q_{0} \otimes_{S} P_{j} \rightarrow P_{j} \rightarrow V_{j} \rightarrow 0 .
$$

Therefore it is of importance to know which reflexive $R$-module $\left(P \otimes_{S} Q\right)^{G}$ is for $P$ and $Q$ in $\mathfrak{B}$. To this end we review some well-known facts about reflexive $R$-modules.

Let $L$ be a reflexive $R$-module. Then it is easily seen that $\operatorname{Hom}_{R}(X, L)$ is $R$-reflexive for all $R$-modules $X$. Hence $X^{*}$ and $X^{* *}$ are reflexive $R$-modules for all $R$-modules $X$, where $X^{*}=\operatorname{Hom}_{R}(X, R)$. Also the canonical morphism $X \rightarrow X^{* *}$ induces isomorphisms $\operatorname{Hom}_{R}\left(X^{* *}, L\right) \rightarrow \operatorname{Hom}_{R}(X, L)$ for all reflexive $R$-modules $L$. This obviously uniquely determines the reflexive $R$-module $X^{* *}$. 
Suppose now that $L_{1}$ and $L_{2}$ are in $\mathfrak{\Omega}$. We want to define the "tensor product" $L_{1} \cdot L_{2}$ of $L_{1}$ and $L_{2}$ in $\Omega$. In general, $L_{1} \otimes_{R} L_{2}$ is not even torsion free, let alone reflexive. So we define $L_{1} \cdot L_{2}$ to be $\left(L_{1} \otimes_{R} L_{2}\right)^{* *}$. Now for each reflexive $R$-module $L$, we know that the natural morphism $L_{1} \otimes_{R} L_{2} \rightarrow L_{1} \cdot L_{2}$ induces an $R$-isomorphism $\operatorname{Hom}_{R}\left(L_{1} \cdot L_{2}, L\right) \stackrel{\sim}{\rightarrow} \operatorname{Hom}_{R}\left(L_{1} \otimes_{R} L_{2}, L\right)$ which is functorial in $L_{1}, L_{2}$ and $L$. Also we have the usual $R$-isomorphisms

$$
\operatorname{Hom}_{R}\left(L_{1} \otimes_{R} L_{2}, L\right) \rightarrow \operatorname{Hom}_{R}\left(L_{1}, \operatorname{Hom}_{R}\left(L_{2}, L\right)\right)
$$

which are functorial in $L_{1}, L_{2}$ and $L$. Hence we obtain the $R$-isomorphisms of reflexive $R$-modules $\operatorname{Hom}_{R}\left(L_{1} \cdot L_{2}, L\right) \rightarrow \operatorname{Hom}_{R}\left(L_{1}, \operatorname{Hom}_{R}\left(L_{2}, L\right)\right)$ which are functorial in $L_{1}, L_{2}$ and $L$. It is for this reason that $L_{1} \cdot L_{2}$ is called the "tensor product" of $L_{1}$ and $L_{2}$ in $\Omega$. With these observations in mind, we prove the following

Lemma 3.1. Let $P$ and $Q$ be projective $S[G]$-modules. Then we have the following:

(a) $\operatorname{Hom}_{S}(P, Q)^{G}=\operatorname{Hom}_{R}\left(P^{G}, Q^{G}\right)$;

(b) $\left(P \otimes_{S} Q\right)^{G}=P^{G} \cdot Q^{G}$.

Proof. (a) $\operatorname{Hom}_{S}(P, Q)^{G}=\operatorname{Hom}_{S[G]}(P, Q) \stackrel{\sim}{\rightarrow} \operatorname{Hom}_{R}\left(P^{G}, Q^{G}\right)$ since the fixed point functor $\mathfrak{B} \rightarrow \mathfrak{L}$ is an equivalence of categories.

(b) Let $L$ be in $\&$ and let $U$ be a projective $S[G]$-module such that $U^{G}=L$. Now

$$
\begin{aligned}
\operatorname{Hom}_{R}((P & \left.\left.\otimes_{S} Q\right)^{G}, U\right)=\operatorname{Hom}_{R}\left(\left(P \otimes_{S} Q\right)^{G}, U^{G}\right) \\
& =\operatorname{Hom}_{S[G]}\left(P \otimes_{S} Q, U\right)=\operatorname{Hom}_{S[G]}\left(P, \operatorname{Hom}_{S}(Q, U)\right) \\
& =\operatorname{Hom}_{R}\left(P^{G}, \operatorname{Hom}_{S[G]}(Q, U)\right)=\operatorname{Hom}_{R}\left(P^{G}, \operatorname{Hom}_{R}\left(Q^{G}, U^{G}\right)\right) \\
& =\operatorname{Hom}_{R}\left(P^{G}, \operatorname{Hom}_{R}\left(Q^{G}, L\right)\right)=\operatorname{Hom}_{R}\left(P^{G} \cdot Q^{G}, L\right) .
\end{aligned}
$$

Hence we have an isomorphism of $R$-modules

$$
\operatorname{Hom}_{R}\left(\left(P \otimes_{S} Q\right)^{G}, L\right) \rightarrow \operatorname{Hom}_{R}\left(P^{G} \cdot Q^{G}, L\right)
$$

which is functorial in $L$. Therefore $\left(P \otimes_{S} Q\right)^{G}=P^{G} \cdot Q^{G}$.

As an immediate consequence of this lemma we have the following

Proposition 3.2. For each $L_{j} \neq R$ we have the following:

(a) $\tau\left(L_{j}\right)=w \cdot L_{j}$;

(b) tensoring the exact sequence $0 \rightarrow w \rightarrow E_{0} \rightarrow R$ with $L_{j}$, we obtain the exact sequence $0 \rightarrow w \cdot L_{j} \rightarrow E_{0} \cdot L_{j} \rightarrow L_{j} \rightarrow 0$ which is the almost split sequence for $L_{j}$.

Proof. Since (a) follows from (b), it suffices to prove (b). We have already seen that the minimal projective $S[G]$-resolution of $V_{j}$ can be obtained by tensoring the minimal projective $S[G]$-resolution of $k$ with $P_{j}$ over $S$ obtaining

$$
0 \rightarrow \tau(S) \otimes_{S} P_{j} \rightarrow Q_{0} \otimes_{S} P_{j} \rightarrow S \otimes_{S} P_{j} \rightarrow k \otimes_{S} P_{j}=V_{j} \rightarrow 0 .
$$

Applying the fixed point functor, we obtain the almost split sequence $0 \rightarrow w \cdot L_{j} \rightarrow$ $E_{0} \cdot L_{j} \rightarrow L_{j} \rightarrow 0$, giving our desired result.

As an immediate consequence of this result we have the following slightly different description of the $A R$ quiver of $\mathfrak{L}$.

COROLLARY 3.3. In the AR quiver of $\mathfrak{Q}$ we have that the number of arrows from $L_{i}$ to $L_{j}$ is the multiplicity of $L_{i}$ in the decomposition of $E_{0} \cdot L_{j}$ into indecomposable modules. 
4. Algebras with finitely many indecomposable reflexive modules. Suppose $\mathbf{C}$ is the complex numbers, $G$ a finite subgroup of $\operatorname{GL}(2, \mathbf{C})$ such that the induced linear action on $S=\mathrm{C}[[X, Y]]$ has the property that every height one prime in $R=S^{G}$ is unramified in $S$, or equivalently, $G$ has no pseudo-reflections. Then we know that $\mathfrak{Z}$, the category of reflexive $R$-modules is of finite representation type, i.e. $\mathfrak{Z}$ has only a finite number of nonisomorphic indecomposable modules. In this section we show how the ideas developed in the previous sections can be used to prove the converse, which is also proved in [1] using different techniques. We begin by pointing out some generalizations of results given in the previous sections.

Let $k$ be a field and $S$ an arbitrary local $k$-algebra with maximal ideal $m$ such that $S / \mathrm{m}=k$. Suppose $G$ is a finite group of $k$-automorphisms of $S$ such that the order of $G$ is not divisible by the characteristic of $k$. Then, as before, $\mathrm{m} S[G]$ is the radical of the skew group $\operatorname{ring} S[G]$ and $S[G] / \mathrm{m} S[G]=k[G]$, the ordinary group ring of $G$ over $k$, which is semisimple. As in the previous section, given two $S[G]$-modules $A$ and $B$, we can form the $S[G]$-modules $\operatorname{Hom}_{S}(A, B)$ and $A \otimes_{S} B$. Likewise the argument given before shows that an $S[G]$-module $A$ is projective if and only if $A$ is $S$-free. Therefore $\operatorname{Hom}_{S}(A, B)$ and $A \otimes_{S} B$ are $S[G]$-projective if $A$ and $B$ are $S[G]$-projective.

Proposition 4.1. Let $\mathfrak{B}$ be the category of projective $S[G]$-modules. Then the functor $\mathfrak{B} \rightarrow \bmod k[G]$ given by $P \mapsto P / m P$ for all $P$ in $\mathfrak{B}$ has the following properties:

(a) the natural epimorphism $P \rightarrow P / \mathrm{m} P$ is a projective cover for all $P$ in $\mathfrak{B}$;

(b) $\mathfrak{B} \rightarrow \bmod k[G]$ is full and dense;

(c) $P$ and $Q$ in $\mathfrak{B}$ are isomorphic if and only if the $k[G]-$ modules $P / \mathrm{m} P$ and $Q / \mathrm{m} Q$ are isomorphic;

(d) suppose $P$ and $Q$ are projective $S[G]$-modules; then $P \otimes_{S} Q \mapsto P / \mathrm{m} \otimes_{k} Q / \mathrm{m} Q$ and $\operatorname{Hom}_{S}(P, Q) \mapsto \operatorname{Hom}_{k}(P / \mathrm{m} P, Q / \mathrm{m} Q)$;

(e) $\operatorname{rank}_{S} P=\operatorname{dim}_{k} P / \mathfrak{m} P$ for all $P$ in $\mathfrak{B}$.

Proof. (a) This is a consequence of the fact that the idempotents in $k[G]$ can be lifted to $S[G]$ since the composition $k[G] \rightarrow S[G] \rightarrow k[G]$ is the identity, where the first morphism is the inclusion.

(b) Obvious.

(c) and (d) Same as previous proofs in case $S=k[[X, Y]]$.

(e) Obvious.

As an immediate consequence of this proposition we have the following which we will need in this section:

Corollary 4.2. Let $S$ and $G$ be as above.

(a) Let $V_{0}, V_{1}, \ldots, V_{d}$ be a complete set of nonisomorphic simple $k[G]$-modules and $P_{0}, P_{1}, \ldots, P_{d}$ their projective covers respectively. Then $P_{0}, \ldots, P_{d}$ is a complete set of nonisomorphic indecomposable projective $S[G]$-modules.

(b) $\mathfrak{B}$ is a Krull-Schmidt category, i.e. each $P$ in $\mathfrak{B}$ is isomorphic to a unique sum $\mathrm{L}_{i=0}^{d} n_{i} P_{i}$. 
We now generalize some of the other results in a different direction.

Let $R$ be an integrally closed noetherian domain with field of quotients $K$. Let $L$ be a finite galois extension of $K$ with group $G$ such that $S$, the integral closure of $R$ in $L$, is a finitely generated $R$-module and every height one prime in $R$ is unramified in $S$. Then, as before, the $R$-morphism $S \rightarrow S[G]^{G}$ given by $s \mapsto \sum_{\sigma \in G} \sigma(s) \sigma$ is an isomorphism which we view as an identification. Hence we have the functor $\mathfrak{B} S[G] \rightarrow \operatorname{add}_{R} S$ given by $P \mapsto P^{G}$ for all $P$ in $\mathfrak{P} S[G]$, the category of projective $S[G]$-modules. Also because every height one prime ideal in $R$ is unramified in $S$, we have that the natural $R$-algebra morphism $S[G] \rightarrow \operatorname{End}_{R}(S)$ given by $s \sigma(x)=$ $s(\sigma(x))$ for all $s$ in $S, \sigma$ in $G$ and $x$ in $S$ is an isomorphism [2]. As before, this implies that the natural $R$-algebra morphism $\operatorname{End}_{S[G]}(S[G]) \rightarrow \operatorname{End}_{R}(S)$ given by $f \mapsto f \mid S$ for all $f$ in $\operatorname{End}_{S[G]}(S[G])$ is an isomorphism. This yields the following result:

Proposition 4.3. The functor $\mathfrak{B} S[G] \rightarrow \operatorname{add}_{R} S$ given by $P \mapsto P^{G}$ for all $P$ in $\mathfrak{B} S[G]$ is an equivalence of categories.

As before, $S$ is a reflexive $R$-module. Suppose $L_{1}$ and $L_{2}$ are reflexive $R$-modules. Then we have that $\operatorname{Hom}_{R}\left(L_{1}, L_{2}\right)$ is a reflexive $R$-module. Also, as before, we define the tensor product $L_{1} \cdot L_{2}$ of $L_{1}$ and $L_{2}$ in the category of reflexive $R$-modules by $L_{1} \cdot L_{2}=\operatorname{Hom}_{R}\left(\operatorname{Hom}_{R}\left(L_{1} \otimes_{R} L_{2}, R\right), R\right)$. Again, given two $S[G]$-modules $A$ and $B$, we consider $\operatorname{Hom}_{S}(A, B)$ and $A \otimes_{S} B$ as $S[G]$-modules in the usual way. Moreover, $\operatorname{Hom}_{S}(A, B)$ and $A \otimes_{S} B$ are projective $S[G]$-modules if $A$ and $B$ are projective $S[G]$-modules since an $S[G]$-module $X$ is projective is and only if $X$ is a projective $S$-module.

As before we have the following

Proposition 4.4. The functor $\mathfrak{B}[G] \rightarrow \operatorname{add}_{R} S$ has the following properties for $P$ and $Q$ in $\mathfrak{B} S[G]$ :

(a) $\operatorname{Hom}_{S}(P, Q) \mapsto \operatorname{Hom}_{R}\left(P^{G}, Q^{G}\right)$;

(b) $P \otimes_{S} Q \mapsto P^{G} \cdot Q^{G}$.

As an immediate consequence of Proposition 4.4 we have the following

Corollary 4.5. Let $L_{1}, L_{2}$ be in $\operatorname{add}_{R} S$. Then $\operatorname{Hom}_{R}\left(L_{1}, L_{2}\right)$ and $L_{1} \cdot L_{2}$ are in $\operatorname{add}_{R} S$.

For the rest of this section we assume we have a fixed algebraically closed field $k$. By a $k$-algebra $T$ we will always mean a $k$-algebra which is a complete integrally closed noetherian local domain with residue field $k$. As a consequence of the above discussion we have the following

THEOREM 4.6. Let $R$ be a k-algebra with field of quotients $K$. Let $S$ be the integral closure of $R$ in a finite galois extension $L$ of $K$ with galois group $G$ whose order is not divisible by the characteristic of $k$. Moreover, suppose every height one prime in $R$ is unramified in $S$. Then the functors $\mathfrak{B}[G] \rightarrow \operatorname{add}_{R} S$ given by $P \mapsto P^{G}$ for all $P$ in $\mathfrak{P} S[G]$ and $\mathfrak{B} S[G] \rightarrow \bmod k[G]$ given by $P \mapsto P / \mathfrak{m} P$ for all $P$ in $\mathfrak{P} S[G]$ induce the functor $F: \operatorname{add}_{R} S \rightarrow \bmod k[G]$ given by $F(L)=P / m P$, where $P$ is the object in 
$\mathfrak{B} S[G]$ such that $P^{G}=L$. The functor $F: \operatorname{add}_{R} S \rightarrow \bmod k[G]$ has the following properties:

(a) $F$ is full and dense;

(b) $F\left(L_{1}\right) \approx F\left(L_{2}\right)$ if and only if $L_{1} \simeq L_{2}$ for all $L_{1}, L_{2}$ in $\operatorname{add}_{R} S$;

(c) $L$ in $\operatorname{add}_{R} S$ is indecomposable if and only if $F(L)$ is simple in $\bmod k[G]$;

(d) for all $L_{1}, L_{2}$ in $S$, we have $F\left(\operatorname{Hom}_{R}\left(L_{1}, L_{2}\right)\right)=\operatorname{Hom}_{k}\left(F\left(L_{1}\right), F\left(L_{2}\right)\right)$ and $F\left(L_{1} \cdot L_{2}\right)=F\left(L_{1}\right) \otimes_{k} F\left(L_{2}\right)$;

(e) $F(R)=k$, the trivial representation;

(f) $\operatorname{rank}_{R} L=\operatorname{dim}_{k} F(L)$ for all $L$ in $\operatorname{add}_{R} S$.

Corollary 4.7. Under the same hypothesis as Theorem 4.6, the functor $F: \operatorname{add}_{R} S$ $\rightarrow \bmod k[G]$ induces a bijection between the isomorphism classes of indecomposable objects in $\operatorname{add}_{R} S$ and the isomorphism classes of simple objects in $\bmod k[G]$.

We now apply Corollary 4.7 to prove the result promised in the beginning of this section.

Let $k=\mathrm{C}$, the complex numbers, $R$ a $k$-algebra with field of quotients $K$. Let $\Omega$ be the algebraic field extension of $K$ generated by all finite field extensions $L$ of $K$ such that the integral closure $S$ of $R$ in $L$ has the property that the height one prime ideals of $R$ in $S$ are unramified. Then $\Omega$ is galois over $K$.

As an application of Corollary 4.7 we have the following

Proposition 4.8. Let $K \subset L \subset L^{\prime} \subset \Omega$ be field extensions of $K$. Suppose $K \subset L$ and $K \subset L^{\prime}$ are finite galois extensions with galois groups $G$ and $G^{\prime}$, respectively. Suppose $S^{\prime} \supset S \supset R$ are the integral closures of $R$ in $L^{\prime}$ and $L$, respectively. Then $\operatorname{add}_{R} S^{\prime} \supset \operatorname{add}_{R} S$ and they are equal if and only if $S=S^{\prime}$.

Proof. Since $\mathbf{C} \subset R$, the usual trace argument shows that $S$ is an $S$-summand of $S^{\prime}$. Hence $\operatorname{add}_{R} S^{\prime} \supset \operatorname{add}_{R} S$. Now suppose $\operatorname{add}_{R} S^{\prime}=\operatorname{add}_{R} S$. Then $\operatorname{add}_{R} S$ and $\operatorname{add}_{R} S^{\prime}$ have the same number of isomorphism classes of indecomposable $R$-modules. Therefore by Corollary 4.7 we have that $\mathbf{C}[G]$ and $\mathbf{C}\left[G^{\prime}\right]$ have the same number of isomorphism classes of simple modules. Hence $G$ and $G^{\prime}$ have the same number of conjugacy classes. But this implies that $G=G^{\prime}$, since $G=G^{\prime} / H$ where $H$ is the normal subgroup of $G^{\prime}$ consisting of the elements of $G$ leaving $L$ fixed. Therefore $S=S^{\prime}$.

We now prove our desired result.

THEOREM 4.9. Suppose $\operatorname{dim} R=2$ and the number of isomorphism classes of indecomposable reflexive $R$-modules is finite. Then

(a) $\Omega$ is a finite galois extension of $K$ with group $G$;

(b) the integral closure $S$ of $R$ in $\Omega$ is regular, $S^{G}=R$ and we can choose analytically independent elements $X$ and $Y$ is $S$ such that $S=\mathrm{C}[[X, Y]]$ and the action of $G$ on $\mathrm{C}[[X, Y]]$ is linear.

Proof. (a) Suppose $\Omega$ is not a finite extension of $K$. Then there is an infinite proper ascending chain

$$
K \subsetneq L_{1} \varsubsetneqq L_{2} \varsubsetneqq \cdots \subset \Omega
$$


of galois extensions of $K$. Letting $S_{i}$ be the integral extension of $R$ in $L_{i}$, we obtain the infinite proper ascending chain $R \subsetneq S_{1} \subsetneq S_{2} \subsetneq \cdots$. Therefore, by Proposition 4.8 we obtain the infinite proper ascending chain add $S_{1} \subsetneq$ add $S_{2} \subsetneq \cdots$. Since each of the $S_{i}$ is a reflexive $R$-module, this contradicts the fact that there is only a finite number of isomorphism classes of indecomposable reflexive $R$-modules.

(b) Let $S$ be the integral closure of $R$ in $\Omega$. Then $S$ satisfies the purity of the branch locus, i.e. if $L \supset \Omega$ is a finite field extension of $\Omega$ such that the height one primes of $\mathfrak{p}$ in $S$ are unramified in the integral closure $S^{\prime}$ of $S$, then $S$ is unramified in $S^{\prime}$ or equivalently $S=S^{\prime}$. Hence by Mumford we know that $S \simeq \mathrm{C}[[X, Y]]$.

Clearly $S^{G}=R$. The rest of the theorem follows from the fact that since $G$ is finite the action of $G$ on $\mathbf{C}[X, Y]]$ is similar to a linear action.

5. Reflexive splitting trace modules. In this section we introduce the notion of a reflexive splitting trace module which plays an essential role in the next section where we show to what extent the method of constructing almost split sequences for complete two-dimensional quotient singularities described in $\S 3$ can be generalized to arbitrary two-dimensional complete local integrally closed domains. We begin with some generalities.

Throughout this section $R$ denotes a noetherian, integrally closed domain with field of quotients $K$ and all $R$-modules are finitely generated. Suppose $M$ is a torsion free $R$-module. Then $\operatorname{End}_{R}(M) \subset$ End $_{K}\left(K \otimes_{R} M\right)$ and the usual trace map $t$ : End $_{K}\left(K \otimes_{R} M\right) \rightarrow K$ has the property that $t\left(\operatorname{End}_{R}(M)\right) \subset R$ since $R$ is integrally closed. We now point out some important facts concerning the trace $t: \operatorname{End}_{R}(M) \rightarrow$ $R$ which we will need in our discussion of almost split sequences.

Proposition 5.1. Let $R$ be a local ring with maximal ideal $\mathrm{m}$ and $M$ a torsion free $R$-module. Then $t: \operatorname{End}_{R}(M) \rightarrow R$ has the property that $t\left(\operatorname{rad} \operatorname{End}_{R} M\right) \subset \mathrm{m}$.

The proof of this fact goes in several steps.

LEMMA 5.2. Let $R$ be a local domain (not necessarily integrally closed) with field of quotient $K$. Suppose $M$ is a torsion free $R$-module and $f: M \rightarrow M$ an $R$-morphism such that $f^{k}(M) \subset \mathfrak{m} M$ for some $k \geqslant 0$. Further suppose that $x$ in $R$ is an eigenvalue for $f \otimes_{R} K: K \otimes_{R} M \rightarrow K \otimes_{R} M$. Then $x$ is in $\mathrm{m}$.

Proof. Suppose $v$ is a nonzero element of $K \otimes_{R} M$ such that $(K \otimes f)(v)=x v$. Then $v=y / s$ with $y$ in $M$ and $s \in R-\{0\}$, from which it follows that $f(y)=x y$. Since $y \neq 0$, there is a $j$ such that $y$ is not in $\mathrm{m}^{j} M$. But $x^{k+(j-1)} y=f^{k+(j-1)}(y)$ is in $\mathrm{m}^{j} M$. Therefore $x$ is not a unit, i.e. $x$ is in $m$.

Proposition 5.3. Let $R$ be an integrally closed local domain with maximal ideal $m$ and field of quotient $K$. Let $M$ be a torsion free $R$-module and $f: M \rightarrow M$ an $R$-endomorphism with $f^{k}(M) \subset \mathfrak{m} M$ for some $k \geqslant 0$. Then the characteristic polynomial $g(X)=X^{n}+a_{1} X^{n-1}+\cdots+a_{n}$ of $K \otimes_{R} f: K \otimes_{R} M \rightarrow K \otimes_{R} M$ has the property that $a_{i}$ is in $\mathrm{m}$ for all $i \geqslant 1$. In particular, $t(f)=a_{1}$ is in $\mathrm{m}$. 
Proof. Suppose $u_{1}, \ldots, u_{n}$ are the roots of $g(X)$ in some finite field extension of $K$. Since $g(X)$ is in $R[X]$, the $u_{i}$ are integral over $R$, and hence $R\left[u_{1}, \ldots, u_{n}\right]$ is a finitely generated $R$-module because $R$ is noetherian. Therefore $R\left[u_{1}, \ldots, u_{n}\right]$ is noetherian. Let $\mathrm{m}^{\prime}$ be a maximal ideal of $R\left[u_{1}, \ldots, u_{n}\right]$ and let $S=R\left[u_{1}, \ldots, u_{n}\right]_{\mathrm{m}}$ ' Then $S$ is a local noetherian domain with maximal ideal $\mathrm{m}^{\prime} S$ having the property that $\mathfrak{m}^{\prime} S \cap R=\mathfrak{m}$ and $\mathfrak{m} S$ is $\mathfrak{m}^{\prime} S$-primary. Let $L$ be the field of quotients of $S$ and let $S M$ be the $S$-submodule of $L \otimes_{R} M$ generated by $M$. Then $L \otimes f: L \otimes_{R} M \rightarrow L$ $\otimes_{R} M$ has the property that $(L \otimes f)(S M) \subset S M$ and $(L \otimes f)^{k} \subset\left(\mathrm{m}^{\prime} S\right) S M$. Since $g(X)$ is also the characteristic polynomial for $L \otimes f$, it follows that the $u_{1}, \ldots, u_{n}$ are the eigenvalues for $L \otimes f$. Hence, by our previous lemma, the $u_{1}, \ldots, u_{n}$ are in $\mathrm{m}^{\prime} S$. Since the $a_{i}$ are in $R$ and are sums of products of the $u_{i}$, they are in $\mathrm{m}^{\prime} S \cap R=\mathrm{m}$, giving our desired result.

We now return to the proof of Proposition 5.1. Since $\Lambda=\operatorname{End}_{R}(M)$ is a finitely generated $R$-module, we have that $\Lambda / \mathrm{m} \Lambda$ is a finite dimensional $R / \mathrm{m}$-algebra. Hence $(\operatorname{rad} \Lambda)^{n} \subset \mathfrak{m} \Lambda$ for some $n>0$. Therefore if $f: M \rightarrow M$ is in $\operatorname{rad}$ End $M$, then we have that $f^{k}(M) \subset \mathrm{m} M$ for some $k>0$. Hence by Proposition 5.3 we have that $t(f) \in \mathfrak{m}$, which finishes the proof of Proposition 5.1.

Of particular interest to us in this section are the reflexive modules $M$ over an integrally closed domain $R$ having the property that $R$ is isomorphic to a summand of $\operatorname{End}_{R}(M)$ viewed as an $R$-module. We now develop various criteria for $M$ to have this property.

We begin with the following preliminary observation:

Lemma 5.4. Let $M$ be a reflexive $R$-module.

(a) The map $\alpha: \operatorname{End}_{R}(M) \rightarrow \operatorname{Hom}_{R}\left(\operatorname{End}_{R}(M), R\right)$ given by $\alpha(f)(g)=t(f g)$ for all $f$ and $g$ in $\operatorname{End}_{R}(M)$ is an isomorphism of $\operatorname{End}_{R}(M)$ modules.

(b) $\alpha(\mathrm{id})=t$ and so $t$ is a free generator for $\operatorname{Hom}_{R}\left(\operatorname{End}_{R}(M), R\right)$ over $\operatorname{End}_{R}(M)$.

Proof. (a) It is easily checked that $\alpha$ is a morphism of $\operatorname{End}_{R}(M)$-modules which is an isomorphism whenever $M$ is free. Thus

$$
\alpha_{\mathfrak{p}}: \operatorname{End}_{R_{\mathfrak{v}}}(M) \rightarrow \operatorname{Hom}_{R_{\mathfrak{v}}}\left(\operatorname{End}_{R_{\mathfrak{v}}}(M)_{\mathfrak{p}}\right), R_{\mathfrak{p}}
$$

is an isomorphism for all height one prime ideals $\mathfrak{p} \subset R$. The fact that $\alpha$ is an isomorphism now follows from the fact that $\operatorname{End}_{R}(M)$ and $\operatorname{Hom}_{R}(\operatorname{End}(M), R)$ are reflexive $R$-modules and $R$ is integrally closed.

(b) Trivial consequence of (a).

As an easy consequence of this result we have the following

Proposition 5.5. Let $M$ be a reflexive $R$-module. Then $R$ is isomorphic to a summand of $\operatorname{End}_{R}(M)$ (notation: $\left.R \mid \operatorname{End}_{R}(M)\right)$ if and only if the trace $t: \operatorname{End}_{R}(M)$ $\rightarrow R$ is onto.

Proof. Clearly if $t: \operatorname{End}_{R}(M) \rightarrow R$ is onto, then $R \mid \operatorname{End}_{R}(M)$. Suppose $R \mid \operatorname{End}_{R}(M)$. Then there is an $R$-module morphism $f: \operatorname{End}_{R}(M) \rightarrow R$ which is onto. But $f=t \alpha^{-1}(f)$ by Lemma 4. Therefore $t$ is onto. 
For obvious reasons we will say that a reflexive module $M$ over an integrally closed domain $R$ is a splitting trace module if $R \mid \operatorname{End}_{R}(M)$ or equivalently $t\left(\operatorname{End}_{R}(M)\right)=R$.

As an easy consequence of Proposition 5 we have the following

Corollary 5.6. (a) $A$ reflexive $R$-module $M$ is a splitting trace module if rank $M$ is a unit in $R$.

(b) If $R$ contains the rational numbers, then every reflexive $R$-module is a splitting trace module.

Proof. (a) This is a trivial consequence of the fact that $t$ (id) $=\operatorname{rank} M$.

(b) Trivial consequence of (a).

Specializing $R$ a bit, we get the following description of when a reflexive $R$-module is a splitting trace module:

Proposition 5.7. Let $R$ be a complete local ring with maximal ideal $m$ such that $R / \mathrm{m}$ is algebraically closed. Then an indecomposable reflexive $R$-module $M$ is $a$ splitting trace module if and only if $p$, the characteristic of $R / \mathrm{m}$, and rank $M$ are relatively prime when $p \neq 0$.

Proof. Clearly $(p, \operatorname{rank} M)=1$ whenever $p \neq 0$ if and only if rank $M$ is invertible in $R$. Thus if $(p, \operatorname{rank} M)=1$ when $p \neq 0$, then $M$ is a splitting trace module by Corollary 5.6.

Suppose now that $M$ is a splitting trace module. If $p=0$, there is nothing to prove, so suppose $p \neq 0$ and rank $M$ and $p$ are not relatively prime. Then $\operatorname{rank} M \cdot 1$ is in $\mathrm{m}$, the maximal ideal of $R$. Since $R$ is complete and $M$ is indecomposable, we know that $\operatorname{End}_{R}(M)$ is a local ring. Because $R / m$ is algebraically closed we have that $\operatorname{End}_{R}(M) / \operatorname{rad} \operatorname{End}_{R} M=R / m$. Therefore each $f$ in $\operatorname{End}_{R} M$ can be written as a $I+g$ where $I$ is the identity, $a$ is in $R$ and $g$ is in $\operatorname{rad} \operatorname{End}_{R}(M)$. Then $t(f)=a t(I)+t(g)$. Since by Proposition 5.1 we have that $t(g)$ is in $m$ and, by hypothesis, $t(I)=\operatorname{rank} M$ is $m$, it follows that $t(f)$ is in $m$. Hence $t\left(\operatorname{End}_{R}(M)\right) \subset \mathfrak{m}$, which shows that $M$ is not a splitting trace module.

We now want to investigate what $R \mid \operatorname{Hom}_{R}(M, N)$, where $M$ and $N$ are reflexive modules over $R$, means. We begin with the following

LeMMA 5.8. Let $M$ be a reflexive module over $R$ and let $Y$ be a module over $\Gamma=\operatorname{End}_{R}(M)$.

(a) The trace map $t: \Gamma \rightarrow R$ induces an $R$-isomorphism $t_{Y}: \operatorname{Hom}_{\Gamma}(Y, \Gamma) \rightarrow$ $\operatorname{Hom}_{R}(Y, R)$.

(b) Suppose now that $R$ is a complete local ring and $M$ is indecomposable.

If $R$ is an $R$-summand of $Y$, then $\Gamma$ is a $\Gamma$-summand of $Y$.

Proof. (a) The $\Gamma$-isomorphism $\alpha: \Gamma \rightarrow \operatorname{Hom}_{R}(\Gamma, R)$ given $\alpha(f)(g)=t(f g)$ for all $f, g$ in $\Gamma$ induces an isomorphism

$$
\operatorname{Hom}_{\Gamma}(Y, \Gamma) \stackrel{(Y, \alpha)}{\rightarrow} \operatorname{Hom}\left(Y, \operatorname{Hom}_{R}(\Gamma, R)\right)
$$


Also we have the standard isomorphism of $R$-modules $v: \operatorname{Hom}_{\Gamma}\left(Y, \operatorname{Hom}_{R}(\Gamma, R)\right) \rightarrow$ $\operatorname{Hom}_{R}(Y, R)$ given by $v(f)(y)=f(y)(1)$ for all $f$ in $\operatorname{Hom}_{\Gamma}\left(Y, \operatorname{Hom}_{R}(\Gamma, R)\right), y$ in $Y$. Since $t_{Y}=v(Y, \alpha)$, as can easily be checked, we have our desired result that $t_{Y}$ is an $R$-isomorphism.

(b) If $\Gamma$ is not a $\Gamma$-summand of $Y$, then for each $\Gamma$-morphism $f: Y \rightarrow \Gamma$ we have that $\operatorname{Im} f \subset \operatorname{rad} \Gamma$ since $\Gamma$ is a local ring. Since $t: \Gamma \rightarrow R$ has the property that $t(\operatorname{rad} \Gamma) \subset \operatorname{rad} R$, we have by (a) that all $R$-morphisms $g: Y \rightarrow R$ have $\operatorname{Im} g \subset \operatorname{rad} R$. Therefore $R$ is not an $R$ summand of $Y$.

We now apply this lemma to prove the following

Lemma 5.9. Let $M, X, Y$ be reflexive $R$-modules and suppose $\Gamma=\operatorname{End}_{R} M$.

(a) The R-morphism $u: \operatorname{Hom}_{R}(X, Y) \rightarrow \operatorname{Hom}_{\Gamma}{ }^{\text {op }}\left(\operatorname{Hom}_{R}(M, X), \operatorname{Hom}_{R}(M, Y)\right)$, given by $f \mapsto \operatorname{Hom}_{R}(M, f)$ for all $f$ in $\operatorname{Hom}_{R}(X, Y)$, is an isomorphism functorial in $X$ and $Y$, where $\operatorname{Hom}_{R}(M, X)$ is considered an $\Gamma^{\mathrm{op}}$-module by means of the operation of $\Gamma$ on $M$.

(b) The R-morphism u: $\operatorname{Hom}_{R}(X, Y) \rightarrow \operatorname{Hom}_{\Gamma}\left(\operatorname{Hom}_{R}(Y, M), \operatorname{Hom}_{R}(X, M)\right)$, given by $f \mapsto \operatorname{Hom}_{R}(f, M)$ for all $f$ in $\operatorname{Hom}_{R}(X, Y)$, is an isomorphism functorial in $X$ and $Y$.

Proof. (a) and (b) Since the morphisms $u$ and $v$ are obviously $R$-morphisms between reflexive $R$-modules it suffices to prove that they are isomorphisms when localized at height one prime ideals. But if $\mathfrak{p}$ is a height one prime, then $M_{\mathfrak{p}}, X_{\mathfrak{p}}, Y_{\mathfrak{p}}$ are free $R_{p}$-modules, from which it follows that $u_{\mathfrak{p}}$ and $v_{\mathfrak{p}}$ are isomorphisms by induction on the rank of the free $R_{\mathfrak{p}}$-modules $X_{\mathfrak{p}}$ and $Y_{\mathrm{p}}$.

As a consequence of Lemmas 5.8 and 5.9 we obtain the following result which was the main point of this discussion:

Proposition 5.10. Let $M$ and $N$ be two reflexive indecomposable $R$-modules when $R$ is a complete local ring.

(a) If $R \mid \operatorname{Hom}_{R}(N, M)$, then $N \simeq M$ and $M$ is a splitting trace module.

(b) If $M$ is a splitting trace module, then $M \simeq N$ if and only if $R \mid \operatorname{Hom}_{R}(N, M)$.

Proof. (a) Suppose $R \mid \operatorname{Hom}_{R}(N, M)$. Then by Lemma 5.8 we know that there is a $\Gamma$-surjection $j: \operatorname{Hom}_{R}(N, M) \rightarrow \Gamma$ where $\Gamma=\operatorname{End}_{R}(M)$. Then by Lemma 5.9 we know there is an $R$-morphism $g: M \rightarrow N$ such that $j(f)=f g$ for all $f$ in $\operatorname{Hom}_{R}(N, M)$. Hence there is an $f$ in $\operatorname{Hom}_{R}(N, M)$ such that $f g=1_{M}$. Therefore $g$ : $M \rightarrow N$ is a splittable monomorphism and hence an isomorphism since $N$ is indecomposable.

(b) Trivial consequence of (a).

6. Almost split sequences. Throughout this section we assume that $R$ is a complete two-dimensional integrally closed local domain. Our aim is to demonstrate to what extent the method of computing almost split sequences for reflexive $R$-modules given in $\$ 3$ can be generalized to arbitrary $R$. While it is known that in this context almost split sequences always exist for reflexive $R$-modules under our hypothesis on $R$ [3], this fact will not be used in our discussion here in order to make the paper as self-contained as possible. 
We begin by pointing out that what we called the fundamental exact sequence for complete two-dimensional quotient singularities also exists for any $R$. Recall that the dualizing module for $R$, which we denote by $w$, is $\operatorname{Hom}_{T}(R, T)$, where $T$ is a two-dimensional regular local subring of $R$ such that $R$ is a finitely generated $\Gamma$-module. Then the reflexive $R$-modules are precisely the $R$-modules which are $T$-free. Therefore $X \mapsto \operatorname{Hom}_{T}(X, T)$ gives an exact duality on the category Ref $R$ of reflexive $R$-modules. From this the well-known fact that $\operatorname{Ext}_{R}^{1}(X, w)=0$ for all reflexive $R$-modules $X$ follows easily.

Another well-known property of $w$ is that $\operatorname{Ext}^{2}(R / \mathrm{m}, w) \simeq R / \mathrm{m}$ where $\mathrm{m}$ is the maximal ideal of $R$. Therefore all the exact sequences $0 \rightarrow w \rightarrow E \rightarrow R \rightarrow R / \mathrm{m} \rightarrow 0$ representing nonzero elements of $\operatorname{Ext}_{R}^{2}(R / \mathrm{m}, w)$ are isomorphic. We call any such sequence a fundamental exact sequerice. Using the fact that depth $R / \mathrm{m}=2$, it is not difficult to show that the $E$ in a fundamental exact sequence $0 \rightarrow w \rightarrow E \stackrel{p}{\rightarrow} R \rightarrow$ $R / \mathrm{m} \rightarrow 0$ is reflexive, see [3]. Now the importance of the fundamental exact sequence lies in the fact that given any morphism $f: X \rightarrow R$ of reflexive $R$-modules which is not a splittable epimorphism, or equivalently not an epimorphism, then there is a $g: X \rightarrow E$ such that $f=p g$. The reason for this is that in the pullback exact commutative diagram

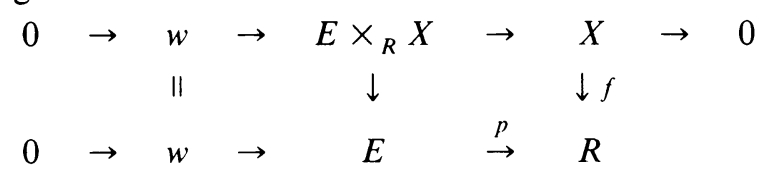

the exact sequence $0 \rightarrow w \rightarrow E \times{ }_{R} X \rightarrow X \rightarrow 0$ splits, giving our desired result.

Proposition 6.1. Let $0 \rightarrow w \rightarrow E \stackrel{p}{\rightarrow} R \rightarrow R / \mathfrak{m} \rightarrow 0$ be the fundamental exact sequence. Then for each reflexive $R$-module $X$ we have that

$$
\operatorname{Im}\left(\operatorname{Hom}_{R}(X, E) \stackrel{\operatorname{Hom}(X, p)}{\rightarrow} \operatorname{Hom}_{R}(X, R)\right)
$$

consists of the nonepimorphisms from $X$ to $R$.

As an immediate consequence of Proposition 6.1, we have the following

COROllaRy 6.2. Let $M$ be a nonprojective indecomposable reflexive $R$-module. Then applying the functor $\operatorname{Hom}_{R}\left(M^{*}\right.$, ) to the fundamental exact sequence $0 \rightarrow w \rightarrow E \stackrel{p}{\rightarrow} R$ $\rightarrow R / \mathrm{m} \rightarrow 0$, we obtain the exact sequence

(*) $\quad 0 \rightarrow \operatorname{Hom}_{R}\left(M^{*}, w\right) \rightarrow \operatorname{Hom}_{R}\left(M^{*}, E\right) \rightarrow M^{* *}=M \rightarrow 0$.

Our aim now is to show that the exact sequence $(*)$ in Corollary 6.2 does not split if and only if $M$ is a splitting trace module. To this end we need the following

LemMA 6.3. Let $M$ be a reflexive $R$-module and $\Gamma=\operatorname{End}_{R}(M)$. Then for each reflexive $R$-module, $X$, the morphism

$$
u_{X}: \operatorname{Hom}_{R}(\Gamma, X) \rightarrow \operatorname{Hom}_{R}\left(M, \operatorname{Hom}_{R}\left(M^{*}, X\right)\right)
$$

given by $\left(u_{X}(f)\right)(m)(g)=f(\delta(g \otimes m))$ for all $f$ in $\operatorname{Hom}_{R}(\Gamma, X), m$ in $M$ and $g$ in $M^{*}$, is an isomorphism of two-sided $\Gamma$-modules which is functorial in $X$ and where $\delta$ : $M^{*} \otimes_{R} M \rightarrow \Gamma$ is given by $\delta(g \otimes m)(x)=g(x) m$ for all $g$ in $M^{*}$ and $m$ and $x$ in $M$. 
Proof. Since $X$ is reflexive, we have that $\delta: M^{*} \otimes_{R} M \rightarrow \Gamma$ induces an isomorphism of two-sided $\Gamma$-modules,

$$
\operatorname{Hom}_{R}(\Gamma, X) \stackrel{\operatorname{Hom}_{R}(\delta, X)}{\rightarrow} \operatorname{Hom}_{R}\left(M^{*} \otimes_{R} M, X\right)
$$

which is functorial in $X$. The standard isomorphism $\alpha_{X}: \operatorname{Hom}_{R}\left(M^{*} \otimes_{R} M, X\right) \rightarrow$ $\operatorname{Hom}_{R}\left(M, \operatorname{Hom}\left(M^{*}, X\right)\right)$ is also a two-sided $\Gamma$-isomorphism functorial in $X$. Our desired result now follows from the fact that $u_{x}=\alpha_{x} \operatorname{Hom}_{R}(\delta, X)$.

We now prove our promised result which we restate as follows:

Proposition 6.4. Let $M$ be an indecomposable reflexive nonprojective $R$-module. Then the following are equivalent:

(a) $M$ is a splitting trace module.

(b) The exact sequence

$$
0 \rightarrow \operatorname{Hom}_{R}\left(M^{*}, w\right) \rightarrow \operatorname{Hom}_{R}\left(M^{*}, E\right) \stackrel{\operatorname{Hom}_{R}\left(M^{*}, p\right)}{\rightarrow} M^{* *} \rightarrow 0
$$

induced from the fundamental exact sequence does not split.

Proof. By the previous lemma we have the following commutative diagram:

$$
\begin{array}{ccc}
\operatorname{Hom}_{R}(\Gamma, E) & \rightarrow & \operatorname{Hom}_{R}(\Gamma, R) \\
\downarrow u_{E} & & \downarrow u_{R} \\
\operatorname{Hom}\left(M, \operatorname{Hom}_{R}\left(M^{*}, E\right)\right) & \rightarrow & \operatorname{Hom}_{R}\left(M, M^{* *}\right) .
\end{array}
$$

Since the $u_{E}$ and $u_{R}$ are isomorphisms, it follows that the top horizontal map is not surjective if and only if the bottom horizontal map is not surjective. Because $\Gamma$ is $R$-reflexive, the basic property of $p: E \rightarrow R$ tells us the top morphism is not surjective if and only if $R \mid \Gamma$ or equivalently if and only if $M$ is a splitting trace module. On the other hand, the bottom row is not surjective if and only if $\operatorname{Hom}_{R}\left(M^{*}, E\right) \rightarrow M^{* *}=M$ is not a splittable epimorphism. Hence we have established the proposition.

Next we want to show that if $M$ is an indecomposable nonprojective $R$-module, then the exact sequence $0 \rightarrow \operatorname{Hom}_{R}\left(M^{*}, w\right) \rightarrow \operatorname{Hom}_{R}\left(M^{*}, E\right) \rightarrow M^{* *} \rightarrow 0$ is almost split if and only if it does not split. Clearly all one has to show is that it is almost split if it does not split. This requires the following preliminary result which has some interest in its own right:

Proposition 6.5. Let $M$ be an indecomposable nonprojective reflexive $R$-module which is a splitting trace module and let $\Gamma=\operatorname{End}_{R} M$. Then

(a) $\operatorname{Hom}_{R}(\Gamma, R) \simeq \Gamma$ as a two-sided $\Gamma$-module.

(b) The two-sided $\Gamma$-morphism

$$
\operatorname{Hom}_{R}(\Gamma, E) \stackrel{\operatorname{Hom}_{R}(\Gamma, p)}{\rightarrow} \operatorname{Hom}_{R}(\Gamma, R)
$$

is not surjective. 
(c) Let $A$ be a $\Gamma$-module which is a reflexive $R$-module, then

$$
\operatorname{Im} \operatorname{Hom}_{\Gamma}\left(A, \operatorname{Hom}_{R}(\Gamma, E)\right) \rightarrow \operatorname{Hom}\left(A, \operatorname{Hom}_{R}(\Gamma, R)\right)
$$

consists of the nonepimorphisms from $A$ to $\operatorname{Hom}_{R}(\Gamma, R)$.

Proof. (a) We have seen that $\operatorname{Hom}_{R}(\Gamma, R)$ is free with generator $t$.

(b) Trivial consequence of $M$ be a splitting trace module.

(c) Let $A$ be a $\Gamma$-module which is a reflexive $R$-module and let $f: A \rightarrow \operatorname{Hom}_{R}(\Gamma, R)$ be a $\Gamma$-morphism. If $f$ is in $\operatorname{Im} \operatorname{Hom}_{R}\left(A, \operatorname{Hom}_{R}(\Gamma, p)\right)$, then $f$ is not an epimorphism since $\operatorname{Hom}_{R}(\Gamma, p)$ is not an epimorphism by (b). Suppose now that $f: A \rightarrow$ $\operatorname{Hom}_{R}(\Gamma, R)$ is not onto. Then for each $a$ in $A$ we have that $f(a)$ is in $\operatorname{rad} \Gamma$. $\operatorname{Hom}_{R}(\Gamma, R)$ and therefore there is $g \in \operatorname{rad} \Gamma$ such that $f(a)=g \cdot t=t \circ g$. Hence $\operatorname{Im} f(a) \subset \mathrm{m}$, the maximal ideal of $R$ by Proposition 5.1 for each $a$ in $A$.

Now we have the commutative diagram

$$
\begin{array}{ccc}
\operatorname{Hom}_{\Gamma}\left(A, \operatorname{Hom}_{R}(\Gamma, E)\right) & \operatorname{Hom}_{\Gamma}\left(A, \operatorname{Hom}_{R}(\Gamma, p)\right) & \operatorname{Hom}_{\Gamma}\left(A, \operatorname{Hom}_{R}(\Gamma, R)\right) \\
\downarrow \phi & & \downarrow \phi^{\prime} \\
\operatorname{Hom}_{R}(A, E) & \stackrel{\operatorname{Hom}_{R}(A, p)}{\rightarrow} & \operatorname{Hom}_{R}(A, R)
\end{array}
$$

where the vertical maps are the standard isomorphisms. Now $\phi(f)(a)=f(a)(1)$ which is in $m$. Therefore $\phi^{\prime}(f)$ is in $\operatorname{Im~}_{\operatorname{Hom}}(A, p)$ which means that $f$ can be lifted to $\operatorname{Hom}_{R}(\Gamma, E)$, which is our desired result.

We are now in position to finish the proof of the following

THEOREM 6.6. Let $0 \rightarrow w \rightarrow E \rightarrow R \rightarrow R / \mathfrak{m} \rightarrow 0$ be a fundamental sequence. Then the following are equivalent for an indecomposable reflexive nonprojective $R$-module $M$ :

(a) $M$ is a splitting trace module.

(b) The induced exact sequence

$$
0 \rightarrow \operatorname{Hom}_{R}\left(M^{*}, w\right) \rightarrow \operatorname{Hom}_{R}\left(M^{*}, E\right) \rightarrow M \rightarrow 0
$$

does not split.

(c) The induced exact sequence

$$
0 \rightarrow \operatorname{Hom}_{R}\left(M^{*}, w\right) \rightarrow \operatorname{Hom}_{R}\left(M^{*}, E\right) \rightarrow M \rightarrow 0
$$

is almost split.

Proof. The equivalence of (a) and (b) has already been shown. Since (c) trivially implies (a), it only remains to show that (a) implies (c).

Let $g: N \rightarrow M$ be a morphism of reflexive modules which is not a splittable epimorphism. Then the induced morphism of $\Gamma^{\mathrm{op}}$-modules $\operatorname{Hom}_{R}(M, f)$ : $\operatorname{Hom}_{R}(M, N) \rightarrow \operatorname{Hom}_{R}(M, M)$ is not surjective. Now we have the commutative diagram of two-sided $\Gamma$-modules

$$
\begin{array}{ccc}
\operatorname{Hom}_{R}\left(M, \operatorname{Hom}_{R}\left(M^{*}, E\right)\right) & \rightarrow & \operatorname{Hom}_{R}(M, M) \\
\downarrow u_{E}^{-1} & & \downarrow u_{R}^{-1} \\
\operatorname{Hom}_{R}(\Gamma, E) & \rightarrow & \operatorname{Hom}_{R}(\Gamma, R)
\end{array}
$$


with vertical isomorphisms. Since the composition of $\Gamma^{\mathrm{op}}$-modules

$$
\operatorname{Hom}(M, N) \rightarrow \operatorname{Hom}_{R}(M, M) \stackrel{u_{R}^{-1}}{\rightarrow} \operatorname{Hom}_{R}(\Gamma, R)
$$

is not surjective, it follows from Proposition 6.5 that it can be lifted to $\operatorname{Hom}_{R}(\Gamma, E)$. It then follows from the above commutative diagram that there is a $\Gamma^{\mathrm{op}}$-morphism $g$ : $\operatorname{Hom}_{R}(M, N) \rightarrow \operatorname{Hom}_{R}\left(M, \operatorname{Hom}\left(M^{*}, E\right)\right)$ such that the diagram of $\Gamma^{\text {op }}$-modules

$$
\begin{array}{rrr} 
& & \operatorname{Hom}_{R}(M, N) \\
& g \swarrow & \downarrow \operatorname{Hom}_{R}(M, f) \\
\operatorname{Hom}_{R}\left(M, \operatorname{Hom}_{R}\left(M^{*}, E\right)\right) \rightarrow & \rightarrow \operatorname{Hom}(M, M)
\end{array}
$$

commutes. But by Lemma 5.9, we know that the functor $\operatorname{Hom}(M):, \operatorname{Ref} R \rightarrow \bmod \Gamma$ given by $X \mapsto \operatorname{Hom}(M, X)$ is fully faithful. Therefore there is a unique

$$
h: N \rightarrow \operatorname{Hom}_{R}\left(M^{*}, E\right)
$$

such that $\operatorname{Hom}_{R}(M, h): \operatorname{Hom}_{R}(M, N) \rightarrow \operatorname{Hom}_{R}\left(M, \operatorname{Hom}_{R}\left(M^{*}, E\right)\right)$ is $g$. Consequently we have that $f: N \rightarrow M$ is the composition

$$
N \stackrel{h}{\rightarrow} \operatorname{Hom}_{R}\left(M^{*}, E\right) \stackrel{\operatorname{Hom}_{R}\left(M^{*}, p\right)}{\rightarrow} M .
$$

Therefore

$$
\operatorname{Hom}_{R}\left(M^{*}, E\right) \stackrel{\operatorname{Hom}_{R}\left(M^{*}, p\right)}{\rightarrow} M
$$

is right almost split. Since this holds for all morphisms of reflexive $R$-modules $f$ : $N \rightarrow M$ which are not splittable epimorphisms and $\operatorname{Hom}_{R}\left(M^{*}, w\right)$ is indecomposable because $M^{*}$ is indecomposable and $\operatorname{Hom}_{R}\left(M^{*}, w\right) \simeq \operatorname{Hom}_{T}\left(M^{*}, T\right)$, it follows that $0 \rightarrow \operatorname{Hom}_{R}\left(M^{*}, w\right) \rightarrow \operatorname{Hom}_{R}\left(M^{*}, E\right) \rightarrow M \rightarrow 0$ is almost split, finishing the proof of the theorem.

We now conclude with some final remarks. For $M$ an indecomposable nonprojective reflexive $R$-module which is a splitting trace module, we have given an independent proof of the existence of an almost split sequence with $M$ at the right-hand end, as well as a method for constructing it. Therefore, if $R$ contains the rational numbers, we have obtained new proof of the existence of almost split sequences for reflexive $R$-modules, as well as a method for constructing them from the fundamental exact sequence. The situation is different when the characteristic of $R / \mathrm{m}$ is $p \neq 0$. While, as noted before, we know there are almost split sequences, the method of constructing almost split sequences from the fundamental sequence given here only proves the existence for indecomposable, nonprojective splitting trace modules.

\section{REFERENCES}

1. M. Artin and J. L. Verdier, Reflexive modules over rational double points, Math. Ann. 270 (1985), 79-82.

2. M. Auslander, On the purity of the branch locus, Amer. J. Math. 84 (1962), 116-125.

3. Fuctors and morphisisms determined by objects, Proc. Conf. Representation Theory (Philadelphia 1976), Dekker, New York, 1978, pp. 1-244. 
4. M. Auslander and J. Carlson, Almost-split sequences and group rings, J. Algebra (to appear).

5. M. Auslander and I. Reiten, Representation theory of artin algebras. III: Almost split sequences, Comm. Algebra 3 (1975), 239-294.

6. G. Gonzalez-Sprinberg and J. L. Verdier, Construction géométrique de la correspondance de McKay, Ann. Sci. Ecole Norm. Sup. 16 (1983), 409-449.

7. J. Herzog, Ringe mit nur endlich vielen Isomorphie-klassen von maximalen unzerlegbaren CohenMacaulay-Moduln, Math Ann. 233 (1978), 21-34.

8. K. W. Roggenkamp and J. Schmidt, Almost split sequences for integral group rings and orders, Comm. Algebra 4 (1976), 693-917.

DePartment of Mathematics, Brandeis University, Waltham, MaSSaChuSETts 02254 